\title{
CONTINUED FRACTIONS WITH SEQUENCES OF PARTIAL QUOTIENTS
}

\author{
K. E. HIRST
}

ABstract. Three results are proved concerning the Hausdorff fractional dimension of sets of continued fractions whose partial quotients belong to given sequences.

Introduction. In [1], I. J. Good investigated the fractional dimension of sets of continued fractions whose partial quotients $a_{n}$ obey various conditions. Included amongst these results are theorems discussing cases where $a_{n}$ becomes large, and in [2] these results were extended to cover some cases where $a_{n}$ tends to infinity rapidly. In all these results the only restrictions on $a_{n}$ are of the type $a_{n} \geqq f(n)$ and $a_{n} \rightarrow \infty$.

In this paper I shall prove analogous results concerning the cases where $a_{n}$ is further restricted to belong to some sequence of natural numbers.

The notation to be used and the relevant parts of the theories of continued fractions and Hausdorff measures are given in [2] and the reader is referred to that paper for these details. In addition throughout the paper we shall use $(\phi n)$ to denote a strictly increasing sequence of natural numbers (rather than $(\phi(n))$-for ease of printing).

The following theorems will be proved.

THEOREM 1. Suppose the series $\sum(\phi n)^{-\alpha}$ converges. Then provided $A$ has the property that

$$
\sum_{\phi n \geqq \Lambda}(\phi n)^{-\alpha} \leqq 2^{-\alpha / 2},
$$

the set $E=\left\{\xi \mid a_{i} \geqq A\right.$ and $\left.a_{i} \in(\phi n)\right\}$ has fractional dimension $\leqq \frac{1}{2} \alpha$.

Theorem 2. Suppose the series $\sum(\phi n)^{-\alpha}$ diverges. Then for any $A$, the set $E$ in Theorem 1 has dimension $\geqq \frac{1}{2} \alpha$.

THEOREM 3. The set

has dimension $1 / 2 b$.

$$
D=\left\{\xi \mid a_{i} \in\left(n^{b}\right) \text { and } a_{i} \geqq i^{b}\right\}
$$

Received by the editors June $26,1972$.

AMS (MOS) subject classifications (1970). Primary 10F20, 10K10; Secondary 28A10.

Key words and phrases. Continued fraction, Hausdorff measure, fractional dimension, partial quotients.

(c) American Mathematical Society 1973 
Proof of Theorem 1. The set $E$ is covered by the system of fundamental intervals $\varepsilon_{n+1}=\left\{I\left(n+1 ; a_{1} \cdots a_{n+1}\right)\right\}$ where $a_{i} \in(\phi n)$ and $a_{i} \geqq A$. Since $\max |I(n)| \rightarrow 0$ as $n \rightarrow \infty, n$ can be chosen so that every member of $\varepsilon_{n+1}$ has length less than $\delta$. We then have

$$
\begin{aligned}
\frac{\left|I\left(n+1 ; a_{1} \cdots a_{n+1}\right)\right|}{\left|I\left(n ; a_{1} \cdots a_{n}\right)\right|} & =\frac{q_{n}\left(q_{n}+q_{n-1}\right)}{q_{n+1}\left(q_{n+1}+q_{n}\right)} \\
& =\frac{q_{n}\left(q_{n}+q_{n-1}\right)}{\left(a_{n+1} q_{n}+q_{n-1}\right)\left(\left(a_{n+1}+1\right) q_{n}+q_{n-1}\right)} \\
& =\frac{1+q_{n-1} / q_{n}}{\left(a_{n+1}+q_{n-1} / q_{n}\right)\left(a_{n+1}+1+q_{n-1} / q_{n}\right)} \leqq \frac{2}{a_{n+1}^{2}} .
\end{aligned}
$$

Thus we have

$$
\sum_{a_{n+1}} \frac{\left|I\left(n+1 ; a_{1} \cdots a_{n+1}\right)\right|^{\alpha / 2}}{\left|I\left(n ; a_{1} \cdots a_{n}\right)\right|^{\alpha / 2}} \leqq 2^{\alpha / 2} \sum_{\phi k \geqq \Delta}(\phi k)^{-\alpha} \leqq 1 .
$$

It follows that

$$
\sum_{\varepsilon_{n+1}}\left|I\left(n+1 ; a_{1} \cdots a_{n+1}\right)\right|^{\alpha / 2} \leqq \sum_{\varepsilon_{n}}\left|I\left(n ; a_{1} \cdots a_{n}\right)\right|^{\alpha / 2} .
$$

Applying this result repeatedly gives, after $n+1$ steps,

$$
\sum_{\varepsilon_{n+1}}|I|^{\alpha / 2} \leqq|I(0)|^{\alpha / 2}=1 .
$$

Thus $L_{\alpha / 2, \delta}(E) \leqq 1$ and so $L_{\alpha / 2}(E)$-the $(\alpha / 2)$-dimensional Hausdorff measure of $E$-does not exceed 1. Therefore $\operatorname{dim} E \leqq \alpha / 2$.

COROLlaRY 1. Let $\alpha$ be the exponent of convergence of the series $\sum(\phi n)^{-1}$. Then if $E^{\prime}=\left\{\xi \mid a_{i} \in(\phi n)\right.$ and $a_{i} \rightarrow \infty$ as $\left.i \rightarrow \infty\right\}, \operatorname{dim} E^{\prime} \leqq \alpha / 2$.

Proof. Let $\beta$ be an arbitrary number greater than $\alpha$. Then $\sum(\phi n)^{-\beta}$ converges. Let $A=A(\beta)$ be specified as in the conditions of Theorem 1 . Since $a_{n} \rightarrow \infty$, there is a natural number $B$ such that for all $n \geqq B, a_{n} \geqq A$. If $E_{I}=\left\{\xi \mid a_{i} \in(\phi n)\right.$ and $a_{i} \geqq A$ for $\left.i \geqq B\right\}$ then $E^{\prime} \subseteq E_{B}$ so that $\operatorname{dim} E^{\prime} \leqq$ $\operatorname{dim} E_{B}$. Also, by the corollary to Good's Lemma 2 [1, p. 206] $\operatorname{dim} E=$ $\operatorname{dim} E_{I 3}$. Thus by Theorem $1, \operatorname{dim} E^{\prime} \leqq \beta / 2$. Since this is true for all $\beta>\alpha$, we have $\operatorname{dim} E^{\prime} \leqq \alpha / 2$.

Proof of Theorem 2. Let $F=\left\{\xi \mid a_{i} \in(\phi n)\right.$ and $\left.A \leqq a_{i} \leqq B\right\}$, where $B$ will be chosen later. We assume without loss of generality that $A$ and $B$ both belong to the sequence $(\phi n)$. Then $E \supseteq F$ so that $\operatorname{dim} E \geqq \operatorname{dim} F$. The set $F$ is closed and bounded, so given an open cover $\mathscr{U}$ of $F$ we can find a finite subsystem of $\mathscr{U}$ which also covers $F$. We discard any intervals of the subsystem which do not meet $F$. The remaining intervals of this 
subsystem may be closed by the addition of their endpoints, and then shrunk so that their endpoints lie in $F$. This gives a system $\mathscr{V}$ of intervals which covers $F$ and which satisfies

$$
\Lambda_{\alpha / 2}(\mathscr{U}) \geqq \Lambda_{\alpha / 2}(\mathscr{V}) .
$$

Note that $F$ contains no isolated points, so that no $J \in \mathscr{V}$ consists of a single point.

We now define another system $\mathscr{W}$ depending on $\mathscr{V}$. Let $J$ be an interval of $\mathscr{V}$. Now $J \subseteq I(0)$, but $J \nsubseteq I(m)$ for $m$ sufficiently large since $\max |I(m)| \rightarrow 0$ as $m \rightarrow \infty$. Thus there is a greatest integer, say $n$, such that $J \subseteq I(n)$. We can therefore find integers $a_{1}, \cdots, a_{n}, k, l$ such that

$$
a_{i} \in(\phi n) \quad \text { for } i=1,2, \cdots, n, \quad k \in(\phi n), l \in(\phi n) ;
$$

$$
\begin{gathered}
A \leqq a_{i} \leqq B \quad \text { for } i=1,2, \cdots, n ; \\
A \leqq k \leqq B, \quad A \leqq l \leqq B, \quad k \neq l ; \\
J \subseteq I(n)=I\left(n ; a_{1} \cdots a_{n}\right),
\end{gathered}
$$

and such that

$$
\begin{aligned}
& J \cap I\left(n+1 ; a_{1} \cdots a_{n} k\right) \neq \varnothing, \\
& J \cap I\left(n+1 ; a_{1} \cdots a_{n} l\right) \neq \varnothing .
\end{aligned}
$$

We now let

$$
K\left(m ; a_{1} \cdots a_{m}\right)=\bigcup_{r=A ; r \in(\phi n)}^{B} I\left(m+1 ; a_{1} \cdots a_{m} r\right) .
$$

Then if $J \supseteq I\left(n+1 ; a_{1} \cdots a_{n} k\right)$ it follows that $J \supseteq K\left(n+1 ; a_{1} \cdots a_{n} k\right)$. If however $J \notin I\left(n+1 ; a_{1} \cdots a_{n} k\right)$ then $J$ must have an endpoint in $I\left(n+1 ; a_{1} \cdots a_{n} k\right)$. But since the endpoints of $J$ belong to $F$, this endpoint must lie in $K\left(n+1 ; a_{1} \cdots a_{n} k\right)$. Thus in both cases we have

$$
J \cap K\left(n+1 ; a_{1} \cdots a_{n} k\right) \neq \varnothing,
$$

and similarly $J \cap K\left(n+1 ; a_{1} \cdots a_{n} l\right) \neq \varnothing$. We therefore have

$$
|J| \geqq \rho\left(K\left(n+1 ; a_{1} \cdots a_{n} k\right), K\left(n+1 ; a_{1} \cdots a_{n} l\right)\right) .
$$

It follows that

$$
|J| \geqq\left|\frac{A\left(k p_{n}+p_{n-1}\right)+p_{n}}{A\left(k q_{n}+q_{n-1}\right)+q_{n}}-\frac{(1+B)\left(l p_{n}+p_{n-1}\right)+p_{n}}{(1+B)\left(l q_{n}+q_{n-1}\right)+q_{n}}\right|
$$

(or this expression with $k$ and $l$ interchanged). On simplification this 
becomes, using the relation $p_{n} q_{n-1}-q_{n} p_{n-1}= \pm 1$,

$$
\begin{aligned}
|J| & \geqq \frac{|(A(1+B)(k-1)+(1+B-A))|}{\left|\left(A\left(k q_{n}+q_{n-1}\right)+q_{n}\right)\left((1+B)\left(l q_{n}+q_{n-1}\right)+q_{n}\right)\right|} \\
& \geqq \frac{|A(1+B)(k-l)+1+B-A|}{A(k+2) q_{n} \cdot 2 B \cdot 2 l\left(q_{n}+q_{n-1}\right)} \\
& \geqq \frac{(A-1) B}{2 A B(k+2) l} \cdot \frac{1}{q_{n}\left(q_{n}+q_{n-1}\right)} \\
& =\frac{(A-1) B \cdot|I(n)|}{2 A B(k+2) l} \geqq \frac{\frac{1}{2} A B|I(n)|}{2 A B \cdot 3 k l} \\
& =\frac{|I(n)|}{12 k l} \geqq \frac{|I(n)|}{12 B^{2}} .
\end{aligned}
$$

We now let $\mathscr{W}$ be the finite set of intervals $I(n)$ corresponding to the intervals $J$ of $\mathscr{V}$. Since a given $I(n)$ in $\mathscr{W}$ may correspond to more than one $J$ in $\mathscr{V}$ we have

$$
\sum|J|^{\alpha / 2} \geqq \sum_{I(n) \in \mathscr{W}} \frac{|I(n)|^{\alpha / 2}}{12^{\alpha / 2} B^{\alpha}} .
$$

We may discard any interval $I(n)$ which is contained in any other interval of $\mathscr{W}$ without altering the fact that our system of fundamental intervals covers $F$ (which it does in virtue of the property $J \subseteq I(n)$ ). Thus we have a finite system $\mathscr{W}$ of fundamental intervals of various orders, none contained in any other. Suppose the largest order of any interval present is $m$. If $m>0$ then $\mathscr{W}$ contains an interval $I\left(m ; a_{1} \cdots a_{m-1} a_{m}\right)$ of order $m$, but no interval of order greater than $m$, and no interval $I(n)$ with $n<m$ for which $I\left(m ; a_{1} \cdots a_{m}\right) \subseteq I(n)$. Since each interval $I\left(m ; a_{1} \cdots a_{m-1} r\right)$ for which $r \in(\phi n)$ and $A \leqq r \leqq B$ contains infinitely many points of $F$, these intervals must all be members of $\mathscr{W}$ (since any two have at most one point in common). We now replace these intervals by the interval $I\left(m-1 ; a_{1} \cdots a_{m-1}\right)$ to give a new system $\mathscr{X}$, and we investigate the effect of this. We have

$\sum_{r=A: r \in(\phi n)}^{B} \frac{\left|I\left(m ; a_{1} \cdots a_{m-1} r\right)\right|^{\alpha / 2}}{\left|I\left(m-1 ; a_{1} \cdots a_{m-1}\right)\right|^{\alpha / 2}}$

$$
\begin{aligned}
& =\sum_{r=A: r \in(\phi n)}^{B} \frac{\left(q_{m-1}\left(q_{m-1}+q_{m-2}\right)\right)^{\alpha / 2}}{\left(r q_{m-1}+q_{m-2}\right)^{\alpha / 2}\left((r+1) q_{m}+q_{m-1}\right)^{\alpha / 2}} \\
& =\sum_{r=A ; r \in(\phi n)}^{B} \frac{\left(1+q_{m-2} / q_{m-1}\right)^{\alpha / 2}}{\left(r+q_{m-2} / q_{m-1}\right)^{\alpha / 2}\left(r+1+q_{m-2} / q_{m-1}\right)^{\alpha / 2}} \\
& \geqq \sum_{r=A ; r \in(\phi n)}^{B} \frac{1}{(r+1)^{\alpha / 2}(r+2)^{\alpha / 2}} \geqq \sum_{r=A ; r \in(\phi n)}^{B} \frac{1}{6^{\alpha / 2} r^{\alpha}} .
\end{aligned}
$$


Thus we have

$$
\begin{aligned}
\sum_{r=A: r \in(\phi n)}^{B}\left|I\left(m ; a_{1} \cdots a_{m-1} r\right)\right|^{\alpha / 2} & \\
& \geqq\left|I\left(m-1 ; a_{1} \cdots a_{m-1}\right)\right|^{\alpha / 2} 6^{-\alpha / 2} \sum_{A \leqq \phi n \leqq B}(\phi n)^{-\alpha} .
\end{aligned}
$$

Now given $A, B$ may be chosen so that

$$
6^{-\alpha / 2} \sum_{A \leqq \phi n \leqq B}(\phi n)^{-\alpha} \geqq 1,
$$

since $\sum(\phi n)^{-\alpha}$ is divergent.

Thus (5) holds for the system $\mathscr{X}$. We proceed in this manner and after a finite number of steps we reach a system whose largest order is zero, and for which (5) holds. The only interval of order zero is $[0,1]$ and so we have

$$
\Lambda_{\alpha / 2}(\mathscr{U}) \geqq \sum|J|^{\alpha / 2} \geqq 12^{-\alpha / 2} B^{-\alpha} \text {. }
$$

This is a positive constant independent of the system $\mathscr{U}$, and so we have shown that $\operatorname{dim} F \geqq \alpha / 2$.

COROLlaRY 2. Let $\alpha$ be the exponent of convergence of the series $\sum(\phi n)^{-1}$. Then if

$$
F=\left\{\xi \mid a_{n} \in(\phi n) \text { and } a_{n} \geqq A\right\},
$$

$\operatorname{dim} F \geqq \alpha / 2$.

If in this corollary the condition $a_{n} \geqq A$ could be replaced by $a_{n} \rightarrow \infty$, this together with Corollary 1 would establish that the set $\left\{\xi \mid a_{n} \in(\phi n)\right.$ and $\left.a_{n} \rightarrow \infty\right\}$ has dimension exactly equal to the exponent of convergence of the series $\sum(\phi n)^{-1}$. At the present time I am unable to prove this for an arbitrary sequence $(\phi n)$, but Theorem 3 gives the result for sequences $\left(n^{b}\right)$.

Proof of TheOREM 3. The arguments are the same as those of Theorem 2 , except that some of the calculations are altered.

We consider the set

$$
D^{\prime}=\left\{\xi \mid a_{i} \in\left(n^{b}\right) \text { and } i^{b} \leqq a_{i} \leqq c i^{b}\right\}
$$

where $c=s^{b}$ will be chosen later. $D^{\prime} \subseteq D$, so that $\operatorname{dim} D^{\prime} \leqq \operatorname{dim} D$. In the proof of Theorem 2, we replace $A$ by $i^{b}$ and $B$ by $c i^{b}$ in (1), (2) and (3). The argument proceeds as before to (4), which becomes

$|J| \geqq\left|\frac{(n+2)^{b}\left(k p_{n}+p_{n-1}\right)+p_{n}}{(n+2)^{b}\left(k q_{n}+q_{n-1}\right)+q_{n}}-\frac{\left(1+c(n+2)^{b}\right)\left(l \dot{p}_{n}+p_{n-1}\right)+p_{n}}{\left(1+c(n+2)^{b}\right)\left(l q_{n}+q_{n-1}\right)+q_{n}}\right|$, 
which on simplification becomes

$$
\begin{aligned}
|J| & \geqq \frac{(n+2)^{b}\left(1+c(n+2)^{b}\right)-1-c(n+2)^{b}-(n+2)^{b}}{(n+2)^{b}(k+2) q_{n} \cdot 2 c(n+2)^{b} \cdot 2 l\left(q_{n}+q_{n-1}\right)} \\
& \geqq \frac{\left((n+2)^{b}-1\right) c(n+2)^{b}|I(n)|}{(n+2)^{b} 3 k 2 c(n+2)^{b} 2 l} \\
& \geqq \frac{\frac{1}{2} c(n+2)^{2 b}}{12 k l c(n+2)^{2 b}}|I(n)| \\
& =\frac{|I(n)|}{24 k l} \geqq \frac{|I(n)|}{24 c^{2}(n+1)^{2 b}} .
\end{aligned}
$$

Thus

$$
\sum|J|^{1 / 2 b} \geqq \sum \frac{|I(n)|^{1 / 2 b}}{24^{1 / 2 b} c^{1 / b}(n+1)} .
$$

The argument proceeds now as from (5) to (6), at which point we have

$$
\begin{aligned}
&\left.\sum_{r=m^{b} ; r \in\left(n^{b}\right)}^{c m^{b}} \frac{\mid I\left(m ; a_{1} \cdots\right.}{\mid I(m-1 ;} a_{1} \cdots a_{m-1} r\right)\left.\right|^{1 / 2 b} \\
&=\sum_{r=m^{b} ; r \in\left(n^{b}\right)}^{c m^{b}} \frac{\left(q_{m-1}\left(q_{m-1}+q_{m-2}\right)\right)^{1 / 2 b}}{\left(r q_{m-1}+q_{m-2}\right)^{1 / 2 b}\left((r+1) q_{m}+q_{m-1}\right)^{1 / 2 b}} \\
& \geqq \sum_{r=m^{b}: r \in\left(n^{b}\right)}^{c m^{b}} 6^{-1 / 2 b} r^{1 / 2 b}=6^{-1 / 2 b} \sum_{t=m}^{s m} \frac{1}{t} \\
& \geqq 6^{-1 / 2 b} \int_{m+1}^{s m} \frac{d t}{t}=6^{-1 / 2 b} \ln \left(\frac{s m}{m+1}\right) \geqq 6^{-1 / 2 b} \ln \left(\frac{1}{2} s\right) .
\end{aligned}
$$

We now choose $s$ so that $6^{-1 / 2 b} \ln \left(\frac{1}{2} s\right) \geqq 2$. We then have

$$
\begin{aligned}
\frac{1}{m} \sum_{r=m^{b}: r \in\left(n^{b}\right)}^{c m^{b}}\left|I\left(m ; a_{1} \cdots a_{m-1} r\right)\right|^{1 / 2 b} & \geqq \frac{2}{m}\left|I\left(m-1 ; a_{1} \cdots a_{m-1}\right)\right|^{1 / 2 b} \\
& \geqq \frac{1}{m-1}\left|I\left(m-1 ; a_{1} \cdots a_{m-1}\right)\right|^{1 / 2 b} .
\end{aligned}
$$

Relation (7) therefore holds for the system of intervals obtained by replacing the intervals $\left\{I\left(m ; a_{1} \cdots a_{m-1} r\right)\right\}$ by the interval $I\left(m-1 ; a_{1} \cdots a_{m-1}\right)$. The argument finishes as before, to establish that $\operatorname{dim} D \geqq 1 / 2 b$. To obtain equality, we note that $D \subseteq\left\{\xi \mid a_{i} \in\left(n^{b}\right)\right.$ and $\left.a_{i} \rightarrow \infty\right\}$ so that $\operatorname{dim} D \leqq 1 / 2 b$ by Corollary 1 .

COROllary 3. The set $\left\{\xi \mid a_{i} \in\left(n^{b}\right)\right.$ and $\left.a_{i} \rightarrow \infty\right\}$ has dimension $1 / 2 b$. 
CONCLUDING REMARKs. It would be interesting to be able to deal with $\left\{\xi \mid a_{i} \in(\phi n)\right.$ and $\left.a_{i} \geqq f(i)\right\}$ for functions $f$ tending to infinity, and I would conjecture that no matter how rapidly $f$ tends to infinity, the set would still have dimension equal to the exponent of convergence of the series $\sum(\phi n)^{-1}$. This would modify Good's Theorem 8 [1, p. 204] to say that the set considered in that theorem has dimension $\geqq \frac{1}{2}$, instead of zero. (I noted in [2] that Dr. Good has kindly confirmed that his proof of Theorem 8 is invalid, but I cannot yet prove either the suggested modification or the general conjecture above.)

\section{REFERENCES}

1. I. J. Good, The fractional dimensional theory of continued fractions, Proc. Cambridge Philos. Soc. 37 (1941), 199-228. MR 3, 75.

2. K. E. Hirst, $A$ problem in the fractional dimension theory of continued fractions, Quart. J. Math. Oxford Ser. (2) 21 (1970), 29-35. MR 41 \#3424.

Department of Mathematics, The University, Highfield, Southampton, ENGLAND 